\title{
Correlation functions at the bulk point singularity from the gravitational eikonal S-matrix
}

\author{
Carlos Cardona \\ Niels Bohr International Academy and Discovery Center, University of Copenhagen, \\ Niels Bohr Institute, Blegamsvej 17, DK-2100 Copenhagen Ø, Denmark \\ E-mail: carlosgiraldo@nbi.ku.dk
}

ABSTRACT: The bulk point singularity limit of conformal correlation functions in Lorentzian signature acts as a microscope to look into local bulk physics in AdS. From it we can extract flat space scattering processes localized in AdS that ultimate should be related to corresponding observables on the conformal field theory at the boundary. In this paper we use this interesting property to propose a map from flat space s-matrix to conformal correlation functions and try it on perturbative gravitational scattering. In particular, we show that the eikonal limit of gravitation scattering maps to a correlation function of the expected form at the bulk point singularity. We also compute the inverse map recovering a previous proposal in the literature.

KeYwords: 1/N Expansion, AdS-CFT Correspondence, Conformal Field Theory, Scattering Amplitudes

ARXIV EPRINT: 1906.08734 


\section{Contents}

1 Introduction and motivation 1

2 Bulk point singularity limit of the four-point correlation function 2

3 Mapping for gravitational dual theories $\quad 6$

$\begin{array}{lll}3.1 & \text { Witten diagrams at the bulk point } & 7\end{array}$

3.1.1 Scalar exchange 8

3.1.2 Graviton exchange $\quad 8$

$\begin{array}{ll}3.1 .3 \text { One loop box } & 10\end{array}$

4 Eikonal resummation $\quad 10$

$\begin{array}{ll}\text { 4.1 Eikonal approximation for the gravitational s-matrix } & 11\end{array}$

$\begin{array}{lll}4.2 & \text { From eikonal s-matrix to the bulk point singularity } & 13\end{array}$

$\begin{array}{llr}5 & \text { Inverse map } & 15\end{array}$

6 Conclusions 16

\section{Introduction and motivation}

According to the AdS/CFT correspondence [1, 2], correlation functions in the boundary conformal field theory are equivalently described through scattering processes in AdS. Since the early days of the conjecture a recurrent question has been how to relate the flat space limit of observables in AdS with correlation functions on the boundary's conformal field theory $[3,4]$. There are several possible ways to achieve this limit and the convenience of each approach depends on the particular problem one would like to explore. An obvious possibility is to think of AdS as a box of size $R$ that should be rescaled to infinite volume $R \rightarrow \infty[5-7]$, in this way we also need to rescale energies and spins of the operators inside the correlator to avoid overrun those scales with the infinite radius of AdS.

One can also use the remarkable similarity between the Mellin representation of conformal correlation functions and flat space s-matrix as a guide line to figure out a proper limit that takes the former into the latter [8-11].

A more recent proposal that have gained some attention over the last couple of years to map scattering amplitudes in flat space to conformal correlation functions in lower dimensions, (even though so far is not completely clear how it connects to AdS/CFT, some discussions towards this connection has been undergone recently, see for example [12-14]), is motivated by the asymptotic BMS symmetries in gravitational theories $[15,16]$, and is based on the observation that the $d$-dimensional conformal symmetry can be linearly realized as a $d+2$-dimensional Lorentz symmetry which in turns allows to write the plane 
waves in flat Minkowskian space as an expansion in conformal primary wave functions [17]. For some progress in this direction see [14, 18-27]

Another approach is to consider scattering processes that under certain conditions are very localized in the middle of AdS and therefore are insensitive to the curvature of the space [3, 4, 28-31]. A suitable Lorentzian configuration to investigate bulk locality is one in which the operators live inside the Milne cone, such as in particular for a four-point correlation function two of the operators insertions are space-like separate and live in the future light-cone of other two space-like separated insertions, as shown in figure 1. By taking the limit where the Graham determinant of the matrix made up from the insertions distances vanish, the correlation function develops a singularity that pick up the local physics in the bulk. This special singularity has been termed "The bulk point singularity" by [32]. In this paper we follow closely the spirit of $[29,30,32]$ and use this particular method in the case of a four point correlation function.

We can in fact have a more general point of view of the bulk point singularity that might allow us to think in applications for theories without a gravity dual. Four-point correlation functions in conformal field theory are usually represented in an operator product expansion (OPE) throughout conformal blocks. Such representation is very reminiscent of the partial wave expansion of the flat space s-matrix. On the other hand, at the bulk point singularity, the conformal blocks are written in terms of spherical harmonic functions, and hence the OPE expansion of the correlation function adopts a similar form as an s-matrix partial wave expansion. In this paper we exploit this similarity to propose a map between these two seemingly unconnected observables. We use this map to compute Witten diagrams at the bulk point singularity from their simpler flat space Feynman diagrams counterpart. By taking advanced of the all-loop resummation of gravitation scattering in flat space at the eikonal approximation, ${ }^{1}$ we can compute the corresponding resummation of the leading singularity expansion of Witten diagrams.

The remaining of this paper is organized as follows, in section 2 we review the bulk point singularity limit of a four-point OPE and show how it exposes the associated flatspace scattering. In section 3 we present a map from the flat-space s-matrix to correlation functions for theories with gravity dual description and test it on the simplest gravitational two-to-two scatterings. In section 4 we apply the aforementioned map to the resummed eikonal gravitational s-matrix. In section 5 we compute the inverse map, namely taking a correlation function to a flat s-matrix and recover a previous conjecture made in [31]. Section 6 contains the conclusions.

\section{Bulk point singularity limit of the four-point correlation function}

We want to consider a four-point correlation function of primary scalar operators which for the sake of simplicity we take of having the same conformal dimension. Up to an overall factor, it is a function of cross-ratios only,

$$
\left\langle\mathcal{O}_{\varphi}\left(x_{1}\right) \mathcal{O}_{\varphi}\left(x_{2}\right) \mathcal{O}_{\varphi}\left(x_{3}\right) \mathcal{O}_{\varphi}\left(x_{4}\right)\right\rangle=\frac{1}{\left(x_{12}^{2}\right)^{\Delta_{\varphi}}\left(x_{34}^{2}\right)^{\Delta_{\varphi}}} A(z, \bar{z})
$$

\footnotetext{
${ }^{1}$ The eikonal approximation in AdS spaces have been studied in a series of interesting papers [33-37].
} 


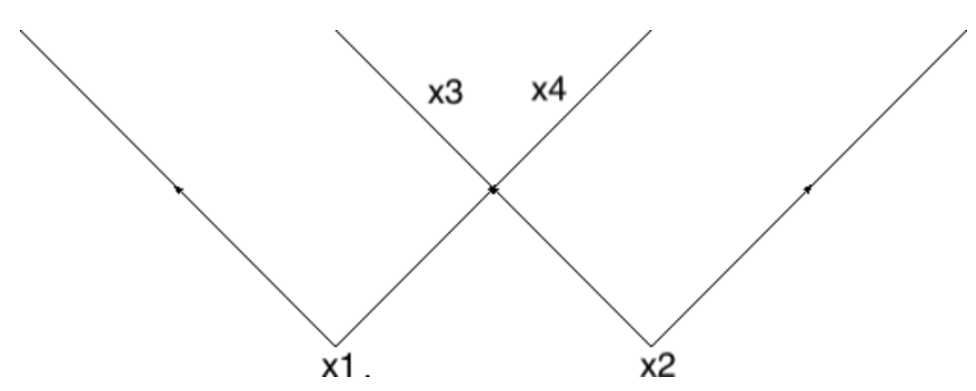

Figure 1. Lorentzian configuration for the bulk point singularity.

where the usual cross-ratios are given by,

$$
z \bar{z}=\frac{x_{12}^{2} x_{34}^{2}}{x_{13}^{2} x_{24}^{2}}, \quad(1-z)(1-\bar{z})=\frac{x_{14}^{2} x_{23}^{2}}{x_{13}^{2} x_{24}^{2}} .
$$

However, we will find convenient to use instead $z=\sigma \mathrm{e}^{\rho}, \bar{z}=\sigma \mathrm{e}^{-\rho}$, where $\sigma$ and $\rho$ can be written in terms of the operator positions as,

$$
\sigma^{2}=\frac{x_{12}^{2} x_{34}^{2}}{x_{13}^{2} x_{24}^{2}}, \quad \sinh ^{2} \rho=\frac{\operatorname{det}\left(x_{i j}^{2}\right)}{4 x_{12}^{2} x_{34}^{2} x_{13}^{2} x_{24}^{2}} .
$$

We then represent the cross-ratios dependence of the correlator in terms of an S-channel operator product expansion,

$$
A(\rho, \sigma)=1+\sum_{\Delta=0}^{\infty} \sum_{\ell=2 n-1} p_{\Delta, \ell} g_{\Delta, \ell}(\rho, \sigma),
$$

We would like to consider the lorentzian configuration depicted at figure 1 and look near the region $\rho \ll 1$. It turns out that in the above given kinematics, the correlator develops a singularity at $\rho=0$ [28] which as been termed as "the bulk point singularity" by [32]. In order to understand the analytic continuation from the Euclidean correlator to the corresponding Lorentzian configuration, it is useful to use the embedding coordinates in $\mathbb{M}^{D+1}$ for euclidean AdS (with $D=d+1$ ),

$$
P_{i}^{E}=\left(\cosh \tau_{i}, \sinh \tau_{i}, \cos \phi_{i}, \sin \phi_{i}, \mathbf{0}^{(d-2)}\right) .
$$

such that the Lorentzian configuration is obtained by the usual Wick rotation in global time $\tau_{E} \rightarrow i \tau$, taking us to the embedding coordinates on $\mathbb{M}^{d+2}$,

$$
P_{i}=\left(\cos \tau_{i}, \sin \tau_{i}, \cos \phi_{i}, \sin \phi_{i}, \mathbf{0}^{(d-2)}\right) .
$$

In order to maintain convergence of the OPE we should take $\tau \rightarrow(\tau-i \xi)$ with $\xi$ small and positive (see [32] for details). Therefore, the Lorentzian configuration represented in 1 corresponds to points located at,

$$
\begin{array}{ll}
\tau_{1}=\tau_{2}=-\pi+i \xi, & \tau_{3}=\tau_{4}=0+i \xi, \\
\phi_{1}=\phi+\pi, \quad \phi_{2}=\phi, & \phi_{3}=0, \quad \phi_{4}=\pi,
\end{array}
$$

The singularity is approached by taking the limit where $\xi \rightarrow 0 .^{2}$

\footnotetext{
${ }^{2}$ See [28] for details on the analytic continuation from the Euclidean correlator to the bulk-point Lorentzian regime.
} 
The cross-ratios in this positions are then given by,

$$
\sigma^{2} \sim \frac{1}{\sin ^{4}\left(\frac{\phi}{2}\right)}, \quad \sinh ^{2} \rho \sim-\frac{\xi^{2} \cos ^{2}\left(\frac{\phi}{2}\right)}{\sin ^{2}\left(\frac{\phi}{2}\right)},
$$

where we have taken the small $\xi$ limit.

It is worth to mention the distinction between the configuration considered here, and the so-called light-cone and Regge configurations. In both of the latter cases, distances $x_{13}$ and $x_{24}$ are taken to be time-like while the remaining distances are all space-like. To achieve the Regge limit, we should take on the last configuration $x_{1}$ going to future null infinite and $x_{3}$ to past null infinite. This different regions can be reach by moving position on the Lorentzian cylinder $(\tau, \phi)$, as it has been detailed explained in [32]. For a nice comparison between the analytic continuation from one region to the other, see [38].

The parametrization (2.7) provides us with a nice picture for the conditions under which the singularity is developed. In order to satisfy $\rho=0$, the $\operatorname{condition} \operatorname{det}\left(P_{i} \cdot P_{j}\right)=0$ should be satisfied. This happens if the vectors $P_{i}$ are linearly dependent or if they form a null manifold. At the particular locations (2.7) the vectors or "momentums" $P_{i}$ are automatically conserved at $\xi=0$ and are orthogonal to the bulk-point $X=\left(0,1, \mathbf{0}^{d}\right)$. However more generally, the matrix $S_{i j} \equiv P_{i} \cdot P_{j}$ being singular, has a zero-eigenvector. Following [32], we can define $n_{i}$ as the null vector obtained from $P_{i}$ by removing the second entry such as they are still orthogonal to the bulk point $X=\left(0,1, \mathbf{0}^{d}\right)$. The entries of the zero-eigenvector can be chosen by

$$
k_{a}=(-1)^{a-1} \operatorname{det}_{a}^{\prime}\left(n_{i, b}\right),
$$

where the tilde means the determinant is taken by removing column $b$. The zeroeigenvector $k_{a}$,

$$
\sum_{a} S_{i a} k_{a}=0
$$

can be used to choose a set of conserved momentum by $p_{i}=k_{a} n_{i}$.

Going back to the conformal partial wave expansion (2.4), at the bulk point singularity regime it has been argued in [30] that it should be dominated by large values of the exchanged operator conformal dimension. An evidence of this is given by taking the bulkpoint singularity limit of a tree-level Witten diagram and notice that its singularity is stronger than the one for an individual conformal block, therefore the singularity of the given diagram must arise from an infinite sum over conformal blocks and so it would be visible from the tail of the sum at large $\Delta$ of the analytically continued partial waves. Hence, in order to use the proper OPE representation we need to analytically continue the euclidean conformal blocks to the lorentzian bulk point kinematics (2.7) and then take the large $\Delta$ limit, while keeping $\xi \Delta$-finite. This has been explicitly done in [32] and we borrow their result here,

$$
g_{\Delta, \ell}(\xi, \phi)=\frac{\mathrm{e}^{-i \pi \Delta} 2^{\frac{1-d}{2}} \ell !}{\sqrt{\pi}(d-2)_{\ell}} \frac{C_{\ell}^{d / 2-1}(\cos \phi)}{|\sin \phi|} \sqrt{\Delta} \xi^{\frac{3-d}{2}} K_{\frac{d-3}{2}}(\Delta \xi) \quad(\Delta \gg 1, \Delta \xi \text { fixed })
$$


Where

$$
\xi \sim \frac{\rho}{\sqrt{1-\sigma}}, \quad \text { and } \quad \sin ^{2}\left(\frac{\phi}{2}\right)=\frac{1}{\sigma} .
$$

The conformal block expansion for the four-point function can be then approximated at the leading singularity as,

$$
A(\xi, \phi)=1+\sum_{\Delta=0}^{\infty} \sum_{\ell} \tilde{p}_{\Delta, \ell} \frac{\mathrm{e}^{-i \pi \Delta} 2^{\frac{1-d}{2}} \ell !}{\sqrt{\pi}(d-2)_{\ell}} \frac{C_{\ell}^{d / 2-1}(\cos \phi)}{|\sin \phi|} \sqrt{\Delta} \xi^{\frac{3-d}{2}} K_{\frac{d-3}{2}}(\Delta \xi) .
$$

where we have denoted $\tilde{p}_{\Delta, \ell}$ as the large $\Delta$ approximation for the OPE coefficients $p_{\Delta, \ell}$. We can see that every individual conformal block develops a singularity $\xi^{\frac{3-d}{2}}$, and therefore in general we might expect a stronger singularity on the four-point correlator since we have to sum an infinity number of blocks, unless some very precise cancellation occurs, as for example for the case of free conformal field theories, which of course does not develop a bulk point singularity.

We can rewrite the correlator in a more illuminating way as,

$$
A(\xi, \phi)=1+\sum_{\Delta=0}^{\infty} \xi^{\frac{3-d}{2}} K_{\frac{d-3}{2}}(\Delta \xi) \frac{\sqrt{\Delta}}{|\sin \phi|}\left(\sum_{\ell} \frac{\mathrm{e}^{-i \pi \Delta} \tilde{p}_{\Delta, \ell} 2^{\frac{1-d}{2}} \ell !}{\sqrt{\pi}(d-2)_{\ell}} C_{\ell}^{d / 2-1}(\cos \phi)\right) .
$$

One immediately recognize that the expression in parentheses looks like an $s$-channel $(d+1)$-flat space partial wave expansion of a s-matrix up to an arbitrary global function $h(\Delta)$. In the following section we will show what this function would be for gravitational dual theories. ${ }^{3}$ We therefore define,

$$
T(\sqrt{s} \equiv \Delta, \cos \phi)=h(\Delta) \sum_{\ell} \mathrm{e}^{-i \pi \Delta} \tilde{p}_{\Delta, \ell} \widetilde{C}_{\ell}^{d / 2-1}(\cos \phi),
$$

where we have make the natural identification of the center of mass energy to the conformal dimension of the operator exchanged $\sqrt{s}=\Delta$ and have normalized the Gegenbauer polynomials in a more convenient way as,

$$
\widetilde{C}_{\ell}^{d / 2-1}(\cos \phi)=\frac{2^{\frac{1-d}{2}} \ell !}{\sqrt{\pi}(d-2)_{\ell}} C_{\ell}^{d / 2-1}(\cos \phi) .
$$

We therefore conjecture the following relation between the flat-space S-matrix and the associated correlation function at the bulk boundary point,

$$
A^{d}(\xi, \phi)=1+\sum_{\Delta=0}^{\infty} \xi^{\frac{3-d}{2}} K_{\frac{d-3}{2}}(\Delta \xi) \frac{\sqrt{\Delta}}{|\sin \phi|} T^{d+1}(\sqrt{s}=\Delta, \cos \phi) .
$$

or more conveniently,

$$
\tilde{A}^{d}(\xi, \phi)=\int_{0}^{\infty} d \Delta \xi^{\frac{3-d}{2}} K_{\frac{d-3}{2}}(\Delta \xi) \frac{\sqrt{\Delta}}{|\sin \phi|} T^{d+1}(\sqrt{s}=\Delta, \cos \phi),
$$

\footnotetext{
${ }^{3}$ Notice that the Gegenbauer is in $d$ therefore expanding an element in $D=d+1$. Similarly as an S-matrix in $D=3+1$ is expanded by Gegenbauer with $d=3$, i.e, Legendre polinomials.
} 
where the identity has been absorbed in the redefinition $\tilde{A}^{d}(\rho, \phi)$ and given the fact that the sum is dominated by large values of $\Delta$ we have replaced it by an integration.

Notice that up to this point we have not made any assumption on the nature of the exchanged operators, other that they have large dimension. Perhaps the most subtle question is if we indeed can interpret the coefficients $\mathrm{e}^{-i \pi \Delta} \tilde{p}_{\Delta, \ell}$ as the corresponding partial wave coefficients for physically sensible flat space s-matrix. If that is the case, we should be able to write it as,

$$
\mathrm{e}^{-i \pi \Delta} \tilde{p}_{\Delta, \ell}=\left(\mathrm{e}^{i \delta_{\ell}(\Delta)}-1\right)
$$

where $\delta_{\ell}$ is the phase shift, which we are taken as complex such as the imaginary part corresponds to the inelasticity.

\section{Mapping for gravitational dual theories}

In this section we would like to go back to (2.14) and specialize it to large $N$ theories with gravity dual in order to explore the bulk point singularity limit for correlations functions corresponding to Witten diagrams by using flat-space s-matrix.

As in $[30,32]$ we will assume that the main contribution to the bulk point limit of this correlators comes from the exchanging of double twist-operators $\mathcal{O}_{n, l} \equiv \mathcal{O}_{\varphi} \partial^{2 n} \partial_{\mu_{1}} \cdots \partial_{\mu_{\ell}} \mathcal{O}_{\varphi}$ with classical dimension $\Delta_{n, \ell}^{\text {class }}=2 \Delta_{\varphi}+2 n+\ell$ and which are present in the operators spectrum of any conformal field theory in $d>2[39,40]$. The resulting computation will prove that this is indeed the case. The OPE coefficients for those double-twist operators, have the following form $[30,41]$

$$
\begin{aligned}
p_{\Delta, \ell}= & \frac{32 \pi^{3 / 2} \Gamma(\Delta-1)(h+l-1) \Gamma(2 h+l-2) \Gamma\left(\frac{l+\Delta}{2}\right) \Gamma\left(\frac{1}{2}(-2 h-l+\Delta+2)\right)}{\Gamma\left(h-\frac{1}{2}\right) \Gamma(l+1) \Gamma\left(\Delta_{\varphi}\right)^{2} \Gamma(\Delta-h) \Gamma\left(\frac{1}{2}(l+\Delta-1)\right) \Gamma\left(-h+\Delta_{\varphi}+1\right)^{2}} \\
& \times \frac{\Gamma\left(\frac{1}{2}(-4 h-l+\Delta+2)+\Delta_{\phi}\right) \Gamma\left(\frac{1}{2}(-2 h+l+\Delta)+\Delta_{\varphi}\right)}{\Gamma\left(\frac{1}{2}(-2 h-l+\Delta+1)\right) \Gamma\left(\frac{1}{2}\left(-l+\Delta-2 \Delta_{\phi}+2\right)\right) \Gamma\left(\frac{1}{2}\left(2 h+l+\Delta-2 \Delta_{\varphi}\right)\right)},
\end{aligned}
$$

which for large $\Delta$ can be approximated as (with $2 h=d)^{4}$

$$
\tilde{p}_{\Delta, \ell}=\Delta^{4 \Delta_{\varphi}-3 h}\left(\frac{16^{h-\Delta_{\varphi}+1}(h+\ell-1) \pi^{3 / 2} \Gamma(2 h+\ell-2)}{\Gamma\left(h-\frac{1}{2}\right) \Gamma(\ell+1) \Gamma\left(\Delta_{\phi}\right)^{2} \Gamma\left(-h+\Delta_{\varphi}+1\right)^{2}}\right) .
$$

We can then rewrite the OPE as,

$$
\begin{aligned}
A(\xi, \phi)= & 1+\mathcal{N}_{a} \sum_{\Delta=0}^{\infty} \xi^{\frac{3-d}{2}} K_{\frac{d-3}{2}}(\Delta \xi) \frac{\sqrt{\Delta}}{|\sin \phi|} \Delta^{4 \Delta_{\varphi}-3 h} \\
& \times \sum_{\ell} \mathrm{e}^{-i \pi \Delta} \frac{(h+\ell-1) \Gamma(2 h+\ell-2)}{\ell !} \widetilde{C}_{\ell}^{d / 2-1}(\cos \phi)
\end{aligned}
$$

\footnotetext{
${ }^{4}$ For large $-N$ conformal field theories, the dimension of the double twist is corrected by a small anomalous dimension $\gamma_{n} / N$. The large contribution to the dimension comes from large $n$ and so we are considering $\Delta \sim n \gg \Delta_{\phi}, \ell$.
} 
with

$$
\mathcal{N}_{a}=\frac{16^{h-\Delta_{\varphi}+1} \pi^{3 / 2}}{\Gamma\left(h-\frac{1}{2}\right) \Gamma\left(\Delta_{\varphi}\right)^{2} \Gamma\left(-h+\Delta_{\varphi}+1\right)^{2}} .
$$

So for large $N$ theories we adopt the following modified map, ${ }^{5}$

$$
\tilde{A}(\xi, \phi)=\mathcal{N}_{a} \int_{0}^{\infty} d \Delta \xi^{\frac{3-d}{2}} K_{\frac{d-3}{2}}(\Delta \xi) \frac{\sqrt{\Delta}}{|\sin \phi|} \Delta^{4 \Delta_{\varphi}-3 h} \Delta^{d-3} T^{(d+1)}(\sqrt{s}=\Delta, \phi),
$$

with

$$
T^{(d+1)}(\sqrt{s}=\Delta, \phi)=\Delta^{3-d} \sum_{\ell} \mathrm{e}^{-i \pi \Delta} \frac{(h+\ell-1) \Gamma(2 h+\ell-2)}{\ell !} \widetilde{C}_{\ell}^{d / 2-1}(\cos \phi) .
$$

The reason why we need a specialized map for the case of theories with gravity dual is that in such case double trace operators contribute at large $N$ at the same order as single trace operators and they are actually the main contribution near the bulk point singularity, whereas for generic theories (meaning theories without a large $N$-expansion) we don't expect double twist operators contributions to dominate over single trace operators at large $\Delta .^{6}$

\subsection{Witten diagrams at the bulk point}

In what follows, we would like to consider very simple flat space s-matrices and check if the map proposed above lead us to the expected results.

The simplest diagram is the one associated to a quartic contact term $T^{(d+1)}=\lambda$, being $\lambda$ the coupling at the vertex. Putting this into the proposed map (3.5) yields,

$$
\tilde{A}(\xi, \phi)=\frac{\mathcal{N}_{a} \lambda}{|\sin \phi|} \int_{0}^{\infty} d \Delta(\Delta)^{4 \Delta_{\varphi}-4}(\Delta \xi)^{\frac{3-d}{2}} K_{\frac{d-3}{2}}(\Delta \xi) .
$$

The type of integral we have to perform has the form,

$$
\begin{aligned}
I(\xi, \phi) & =\int_{0}^{\infty} d x x^{4 \Delta_{\varphi}-4+2 \alpha}(x \xi)^{\frac{3-d}{2}} K_{\frac{d-3}{2}}(x \xi) \\
& =\xi^{-2 \alpha-4 \Delta_{\varphi}+3} \Gamma\left(\alpha+2 \Delta_{\varphi}-\frac{3}{2}\right) 2^{\frac{1}{2}\left(4 \alpha-d+8 \Delta_{\varphi}-7\right)} \Gamma\left(-\frac{d}{2}+\alpha+2 \Delta_{\varphi}\right),
\end{aligned}
$$

leading us to,

$$
\begin{aligned}
\tilde{A}(\rho, \phi) & =\frac{\mathcal{N}_{a} \lambda 2^{\frac{1}{2}\left(-d+8 \Delta_{\varphi}-7\right)}}{|\sin \phi|} \frac{\Gamma\left(2 \Delta_{\varphi}-\frac{3}{2}\right) \Gamma\left(2 \Delta_{\varphi}-\frac{d}{2}\right)}{\xi^{4 \Delta_{\varphi}-3}} \\
& =-i \mathcal{N}_{a} \lambda 2^{\frac{1}{2}\left(-d+8 \Delta_{\varphi}-5\right)} \Gamma\left(2 \Delta_{\varphi}-\frac{d}{2}\right) \frac{\Gamma\left(2 \Delta_{\varphi}-\frac{3}{2}\right) \sigma(1-\sigma)^{2 \Delta_{\varphi}-2}}{\rho^{4 \Delta_{\varphi}-3}} .
\end{aligned}
$$

where we have used

$$
\sin \phi=2 \frac{\sqrt{\sigma-1}}{\sigma}
$$

Which up to an unimportant normalization factor is exactly the behavior of the contact Witten diagram in $A d S_{d+1}$ at the bulk point singularity, as has been shown in [28].

\footnotetext{
${ }^{5}$ Notice that this relation is different from the one proposed in [28] where the correlation function is integrated against some localized kernels.

${ }^{6}$ Excluding free theories and special supersymmetric theories with susy-protected double trace operators.
} 


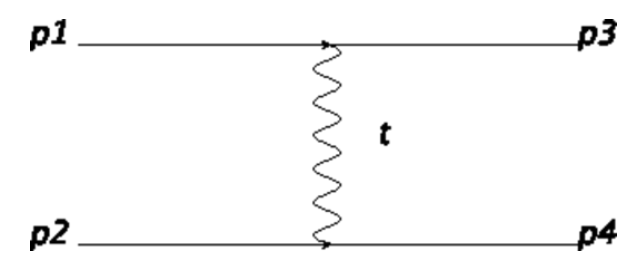

Figure 2. t-graviton exchanged between two scalars.

\subsubsection{Scalar exchange}

As we have discussed in section 2 , the bulk point regime explores large energies $\Delta \gg \Delta_{\varphi},{ }^{7}$ therefore we can approximate the exchange propagator to be massless. A scalar exchange in flat space is given by,

$$
T^{d+1}=\frac{\lambda}{-t}=\frac{\lambda}{\Delta^{2} \sin ^{2} \frac{\phi}{2}},
$$

where we have used,

$$
-\frac{t}{s}=\frac{q^{2}}{s}=\sin ^{2} \frac{\phi}{2}
$$

Putting it back into (3.5), lead us to an integration (3.8) with $\alpha=-1$ resulting in,

$$
\begin{aligned}
\tilde{A}(\rho, \phi) & =\frac{\mathcal{N}_{a} \lambda 2^{\frac{1}{2}\left(-d+8 \Delta_{\varphi}-11\right)}}{|\sin \phi| \sin ^{2} \frac{\phi}{2}} \frac{\Gamma\left(2 \Delta_{\varphi}-\frac{5}{2}\right) \Gamma\left(2 \Delta_{\varphi}-\frac{d}{2}-1\right)}{\xi^{4 \Delta_{\varphi}-5}} \\
& =\frac{\mathcal{N}_{a} \lambda 2^{\frac{1}{2}\left(-d+8 \Delta_{\varphi}-11\right)} \Gamma\left(2 \Delta_{\varphi}-\frac{d}{2}-1\right)}{\sin ^{2} \frac{\phi}{2}} \frac{\Gamma\left(2 \Delta_{\varphi}-\frac{5}{2}\right) \sigma(1-\sigma)^{2 \Delta_{\varphi}-4}}{\rho^{4 \Delta_{\varphi}-5}},
\end{aligned}
$$

which again is the expected behavior of an scalar exchange Witten diagram $[42,43]$ at the bulk point singularity limit.

\subsubsection{Graviton exchange}

Now consider two energetic scalar particles scattering gravitationally in flat space as in figure 2 .

We are going to borrow the expressions from [44] (see details there and references therein). In the case of four-scalars of equal mass $m$ interacting gravitationaly through a single graviton exchange we have,

$$
T^{d+1}=2 i \kappa_{D}^{2} \frac{\gamma(s)}{t}
$$

with

$$
\gamma(s)=\frac{\left(s-2 m^{2}\right)^{2}}{2}-\frac{2}{d-1} m^{4}
$$

and $\kappa_{D}^{2}=8 \pi \mathrm{G}_{d+1}$. In the large $s$-limit,

$$
T^{d+1} \sim i \kappa_{D}^{2} \frac{s^{2}}{t}
$$

\footnotetext{
${ }^{7}$ Even thought we are taken the center of mass energy large, it should be smaller than the characteristic energy where string effects start being relevant.
} 
This expression can as well be easily computed by gluing two three-vertex tree level amplitudes connected by a massless propagator. Namely, for large $s$ there is an effective vertex $\kappa_{D} s$ and the $t$-channel graviton propagator gives a contribution of $\frac{1}{t}$ as shown in figure 2 . By using (3.12) the scattering can be rewritten in terms of the energy as,

$$
T^{d+1}=i \kappa_{D}^{2} \frac{\Delta^{4}}{\Delta^{2} \sin ^{2} \frac{\phi}{2}}=i \kappa_{D}^{2} \frac{\Delta^{2}}{\sin ^{2} \frac{\phi}{2}},
$$

Putting this into (3.5) we have,

$$
\tilde{A}(\xi, \phi)=\frac{\mathcal{N}_{a} \lambda}{|\sin \phi|} \int_{0}^{\infty} d \Delta(\Delta)^{4 \Delta_{\varphi}-2}(\Delta \xi)^{\frac{3-d}{2}} K_{\frac{d-3}{2}}(\Delta \xi) .
$$

leading us to,

$$
\begin{aligned}
A(\rho, \phi) & =\kappa_{d+1}^{2} \mathcal{N}_{a} \frac{1}{|\sin \phi| \sin ^{2} \frac{\phi}{2}} \frac{\Gamma\left(2 \Delta_{\varphi}-\frac{1}{2}\right) \Gamma\left(-\frac{d}{2}+2 \Delta_{\varphi}+1\right)}{\xi^{4 \Delta_{\varphi}-1}} \\
& =\kappa_{d+1}^{2} \mathcal{N}_{a} \frac{\Gamma\left(-\frac{d}{2}+2 \Delta_{\varphi}+1\right)}{\sin ^{2} \frac{\phi}{2}} \frac{\Gamma\left(2 \Delta_{\varphi}-\frac{1}{2}\right) \sigma(1-\sigma)^{2 \Delta_{\varphi}-1}}{\rho^{4 \Delta_{\varphi}-1}} .
\end{aligned}
$$

The corresponding correlator associated to the Witten diagram for a graviton exchange minimally coupled to the external scalars has been computed in [42].

$$
\begin{aligned}
I_{\text {grav }}=\left(\frac{6}{\pi^{2}}\right)^{4}[16 & x_{24}^{2}\left(\frac{1}{2 s}-1\right) D_{4455}+\frac{64}{9} \frac{x_{24}^{2}}{x_{13}^{2}} \frac{1}{s} D_{3355}+\frac{16}{3} \frac{x_{24}^{2}}{x_{13}^{4}} \frac{1}{s} D_{2255} \\
+ & \left.18 D_{4444}-\frac{46}{9 x_{13}^{2}} D_{3344}-\frac{40}{9 x_{13}^{4}} D_{2244}-\frac{8}{3 x_{13}^{6}} D_{1144}\right],
\end{aligned}
$$

where

$$
s \equiv \frac{1}{2} \frac{x_{13}^{2} x_{24}^{2}}{x_{12}^{2} x_{34}^{2}+x_{14}^{2} x_{23}^{2}}
$$

or equivalently,

$$
x_{34}^{2}\left(\frac{1}{2 s}-1\right)=4 \frac{\cos ^{2} \frac{\phi}{2}}{\sin ^{2} \frac{\phi}{2}} .
$$

The dominant contribution at the bulk point singularity, will come from the D-function $D_{4455}$, which behave as (see appendix [28])

$$
D_{\Delta_{\varphi} \Delta_{\varphi} \Delta_{\varphi}+1 \Delta_{\varphi}+1} \sim \Gamma\left(2 \Delta_{\varphi}-\frac{1}{2}\right) \frac{\sigma(1-\sigma)^{2 \Delta_{\varphi}-1}}{\rho^{4 \Delta_{2}-1}}
$$

giving us the following behaviour for the leading bulk-point singularity of the supergravity computation,

$$
I_{\text {grav }} \sim \frac{1}{\sin ^{2} \frac{\phi}{2}} \Gamma\left(2 \Delta_{\varphi}-\frac{1}{2}\right) \frac{\sigma(1-\sigma)^{2 \Delta_{\varphi}-1}}{\rho^{4 \Delta_{\varphi}-1}} .
$$

and which up to a normalization factor, match the result obtained from the flat space s-matrix mapping. 


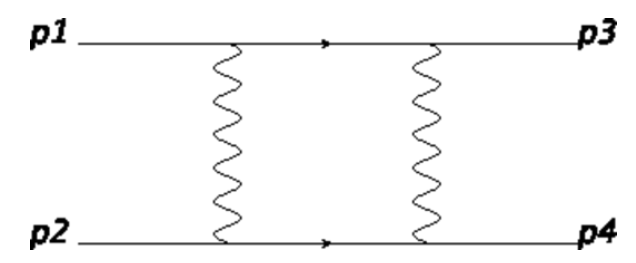

Figure 3. t-two graviton exchanged between two scalars.

\subsubsection{One loop box}

Encouraged by the observations above, we push forward and see if the proposed map translates the perturbative loop expansion in the flat-space scattering to the corresponding perturbative expansion of Witten diagrams at the bulk point limit. For that we now consider the box one-loop contribution at the eikonal limit as shown in figure 3, again borrowing the results from [44].

$$
T_{\square}^{d+1}(s, t)=-\frac{\pi^{\frac{d+5}{2}}}{(2 \pi)^{d+1}} \frac{4 \kappa_{d+1}^{4} \gamma^{2}(s)}{\sqrt{\left(s-2 m^{2}\right)^{2}-4 m^{4}}} \frac{\Gamma\left(\frac{d-3}{2}\right)^{2} \Gamma\left(\frac{5-d}{2}\right)}{\Gamma(d-3)} t^{\frac{d-5}{2}}
$$

which in the large $s$ limit can be taken as,

$$
\begin{aligned}
T_{\square}^{d+1}(s, t) & =-\frac{(-1)^{\frac{d-5}{2}} \pi^{\frac{d+5}{2}}}{(2 \pi)^{d+1}} \kappa_{d+1}^{4} s^{3} \frac{\Gamma\left(\frac{d-3}{2}\right)^{2} \Gamma\left(\frac{5-d}{2}\right)}{\Gamma(d-3)} t^{\frac{d-5}{2}} \\
& =-\frac{\pi^{\frac{d+5}{2}} \kappa_{d+1}^{4}}{(2 \pi)^{d+1}} \frac{\Gamma\left(\frac{d-3}{2}\right)^{2} \Gamma\left(\frac{5-d}{2}\right)}{\Gamma(d-3)} \Delta^{d+1} \sin ^{d-5} \frac{\phi}{2} .
\end{aligned}
$$

putting this back into (3.5) we get

$$
\begin{aligned}
\tilde{A}(\xi, \phi)= & -\frac{\mathcal{N}_{a}}{|\sin \phi| \sin ^{5-d} \frac{\phi}{2}} \frac{\pi^{\frac{d+5}{2}} \kappa_{d+1}^{4}}{(2 \pi)^{d+1}} \frac{\Gamma\left(\frac{d-3}{2}\right)^{2} \Gamma\left(\frac{5-d}{2}\right)}{\Gamma(d-3)} \\
& \times 2^{\frac{1}{2}\left(4 d+8 \Delta_{\varphi}-4\right)} \frac{\Gamma\left(d+1+2 \Delta_{\varphi}-\frac{3}{2}\right) \Gamma\left(\frac{d+1}{2}+2 \Delta_{\varphi}\right)}{\xi^{4 \Delta_{\varphi}-1+2 d}} \\
= & -\frac{\mathcal{N}_{a}}{\sin ^{5-d} \frac{\phi}{2}} \frac{\pi^{\frac{d+5}{2}} \kappa_{d+1}^{4}}{(2 \pi)^{d+1}} \frac{\Gamma\left(\frac{d-3}{2}\right)^{2} \Gamma\left(\frac{5-d}{2}\right)}{\Gamma(d-3)} 2^{\frac{1}{2}\left(4 d+8 \Delta_{\varphi}-4\right)} \Gamma\left(\frac{d+1}{2}+2 \Delta_{\varphi}\right) \\
& \times \Gamma\left(d+1+2 \Delta_{\varphi}-\frac{3}{2}\right) \frac{\sigma(1-\sigma)^{2 \Delta_{\varphi}-1+d}}{\rho^{4 \Delta_{\varphi}-1+2 d}} .
\end{aligned}
$$

At this point we don't know the exact answer from the gravity dual computation, so we can think of this result a prediction for the bulk point singularity limit of a one loop Witten diagram. The naive interpretation of this is that the exponent for the singularity is given by the same as the single-exchange plus a $2 d$ coming from the loop integration.

\section{Eikonal resummation}

By noticing that the bulk point singularity increases with the number of loops, we might wonder if the expansion in loops at the singularity converges. We want to ask the question 
if similarly as in flat space, the sum of loop Witten diagrams in the bulk point limit "exponentiate". In order to study that, we want to use the eikonal approximation of the gravitational flat space s-matrix to compute the corresponding bulk point limit of the correlator.

It is worth to mention here that we will be using the eikonal approximation only as a computable all-loop example of a flat space S-matrix and match it to a corresponding perturbative expansion around the bulk-point region, but we are not trying to relate it with the eikonal approximation in AdS, which is a quite different limit from the bulk point limit and has been considered in great detail previously at [33-37].

\subsection{Eikonal approximation for the gravitational s-matrix}

In this subsection we would like to remind ourselves very quickly the eikonal approximation in gravitation scattering. The eikonal regime, namely $-t / s \ll 1$, turns out to be well described by summing ladder and crossed ladder diagrams, which essentially exponentiates a single-graviton exchange in the so-called phase-shift and in terms of this phase the scattering amplitude is given by the transversal Fourier transform of the resummed graphs, concretely [45-48],

$$
i T_{\text {eik }}(s, t)=2 s \int d^{d-1} \mathbf{b} \mathrm{e}^{-i \mathbf{q} t \cdot \mathbf{b}}\left(\mathrm{e}^{i \chi(b)}-1\right),
$$

Where we denoted by $\mathbf{b}$ the impact parameter and by $\chi$ the phase-shift. This approximation is expected to be valid for relatively large impact parameter withing the following energy region [49],

$$
\left(\mathrm{G}_{d+1} E^{2}\right)^{\frac{1}{d-3}}>b>\left(\mathrm{G}_{d+1} E\right)^{\frac{1}{d-2}},
$$

or equivalently,

$$
\left(\mathrm{G}_{d+1} \Delta^{2}\right)^{\frac{1}{d-3}}>b>\left(\mathrm{G}_{d+1} \Delta\right)^{\frac{1}{d-2}}
$$

and small angles,

$$
\frac{-t}{s}=\sin ^{2} \frac{\phi}{2} \ll 1
$$

$\mathrm{G}_{d+1}$ being the $(d+1)$ - dimensional Newton's gravitational constant. As we mentioned, the leading contribution to the phase-shift at the Eikonal limit is given by the tree level singlegraviton exchange (3.16). From this expression, the phase-shift $\chi$ can be computed by inverting the transverse Fourier transform at equation (4.1) and replacing $T_{\text {eik }}$ with (3.16), resulting in,

$$
\chi(b)=\frac{\kappa_{D}^{2}}{2(D-4) \Omega_{D-3}} \frac{s}{b^{D-4}},
$$

with $D=d+1$. One can notice that the phase shift depends only on the magnitude of the transversal impact parameter vector $\mathbf{b}$ and henceforth when plugging in back into (4.1) we can immediately integrate over angular coordinates, to obtain [48],

$$
i T_{\text {eik }}(s, t)=-\frac{2 i s(2 \pi)^{(d-1) / 2}}{q_{t}^{\frac{d-3}{2}}} \int_{0}^{\infty} d b b^{\frac{d-1}{2}} J_{\frac{d-3}{2}}\left(q_{t} b\right)\left(\mathrm{e}^{i h s / b^{d-3}}-1\right),
$$


where $J_{\nu}(x)$ is the Bessel function of first kind and we have defined $h=\frac{\kappa_{D}^{2}}{2(D-4) \Omega_{D-3}}$ to unclutter the notation. In $d=4$ it simplifies further to,

$$
i T_{\mathrm{eik}}(s, t)=-\frac{2 i s(2 \pi)^{3 / 2}}{q_{t}^{\frac{1}{2}}} \sqrt{\frac{2}{\pi}} \int_{0}^{\infty} d b b \sin \left(q_{t} b\right)\left(\mathrm{e}^{i h s / b}-1\right),
$$

ignoring for now the identity contribution, which will give us the vacuum contribution, we can integrate the exponential,

$$
i T_{\text {eik }}(s, t)=\frac{8(2 \pi)^{3 / 2} h}{\pi} \frac{\Delta^{2}}{\sin \left(\frac{\phi}{2}\right)^{2}} \operatorname{ker}_{2}(\sqrt{2}(1-i) \Theta),
$$

$\operatorname{ker}_{\nu}$ is the Kelvin function defined in terms of the K-Bessel function as,

$$
\operatorname{ker}_{\nu}(z)=\operatorname{Re}\left(\mathrm{e}^{\frac{-\nu \pi i}{2}} K_{\nu}\left(z \mathrm{e}^{\frac{\pi i}{4}}\right)\right)
$$

and we have defined

$$
\Theta=\Delta \sqrt{h \Delta \sin \left(\frac{\phi}{2}\right)} .
$$

In order to reproduce the leading exchanged graviton we should take the argument of the Kelvin function small, i.e $\Theta<1$, and therefore the Kelvin function can be Taylor expanded. The leading term of this expansion is just $1 / 2$ and we recover the single graviton exchange result (3.17).

The eikonal resummation allow us to take the opposite limit as well. For large values of the argument, the Kelvin function behaves as,

$$
\operatorname{ker}_{\nu}(z) \sim \sqrt{\frac{\pi}{2 x}} \mathrm{e}^{\frac{-x}{\sqrt{2}}}\left(f(x) \cos \left(\frac{x}{\sqrt{2}}+\frac{\pi}{8}\right)+g(x) \sin \left(\frac{x}{\sqrt{2}}+\frac{\pi}{8}\right)\right)
$$

where $f(x)$ and $g(x)$ are given as a series expansion in $1 / x$ and whose leading order term is respectively,

$$
f(x) \sim 1, \quad \text { and } \quad g(x) \sim \frac{4 \nu^{2}-1}{8 x \sqrt{2}} .
$$

Therefore, for large argument we can approximate,

$$
\operatorname{ker}_{\nu}(x) \sim \sqrt{\frac{\pi}{2 x}} \mathrm{e}^{\frac{-x}{\sqrt{2}}} \cos \left(\frac{x}{\sqrt{2}}+\frac{\pi}{8}\right)
$$

and the eikonal s-matrix simplifies in this limit to,

$$
i T_{\text {eik }}(s, t)=-2(2 \pi)^{3 / 2} h \frac{\Delta^{2}(i-1)}{\sin \left(\frac{\phi}{2}\right)^{2}} H_{\frac{1}{2}}^{(1)}(2 \Theta),
$$

being $H_{\nu}^{(1)}(2 \Theta)$ the Hankel function of the first kind. Interestingly the amplitude behaves oscillatory for large $\Theta$, but is important to notice that in this ultra energetic regime, we 
expect quantum gravity contributions to start dominating and therefore we should not expect (4.14) to hold.

At this point is worth looking into the regime of validity of the parameters. The eikonal approximation of the s-matrix is valid as long as the impact parameter is larger than the Schwarzschild radius of a mass corresponding to the center of mass energy $b>R_{S}(\Delta) \sim$ $\left(G_{D} \Delta\right)^{1 /(D-3)}$, in other words $\Theta<b^{d-2}$, and as we just mentioned in the paragraph above, $\Theta$ can not be too large.

On the other hand, we also have to satisfy $\xi \Delta \sim \mathcal{O}(1)$ and therefore,

$$
\xi b^{(d-2)}>G_{D}
$$

This means that we should consider a large impact parameter compare to $\xi$, but at the same time we need a large AdS radius such as $b \ll R_{\text {AdS }}$, in order to avoid contributions from the curvature of space time, so the conformal field theory should be strongly coupled.

\subsection{From eikonal s-matrix to the bulk point singularity}

We now want to see how the correlation function looks like at the bulk point singularity as computed from the eikonal flat space result. For that we need to plug (4.6) into (3.5),

$$
\begin{aligned}
\tilde{A}(\xi, \phi)=\frac{2 i(2 \pi)^{(d-1) / 2} \mathcal{N}_{a}}{|\sin \phi|} & \int_{0}^{\infty} d \Delta(\Delta)^{4 \Delta_{\varphi}-4}(\Delta \xi)^{\frac{3-d}{2}} K_{\frac{d-3}{2}}(\Delta \xi) \frac{\Delta^{2}}{\Delta^{\frac{d-3}{2}} \sin ^{\frac{d-3}{2}} \frac{\phi}{2}} \\
& \times \int_{0}^{\infty} d b b^{\frac{d-1}{2}} J_{\frac{d-3}{2}}\left(\Delta \sin \frac{\phi}{2} b\right)\left(\mathrm{e}^{h \Delta^{2} / b^{d-3}}-1\right) .
\end{aligned}
$$

This integral is hard to compute in general dimension, but we can restrict ourselves again to $d=4$, yielding a fairly large expression,

$$
\begin{aligned}
\tilde{A}(\xi, \phi)= & \frac{(2 \pi)^{3 / 2} \mathcal{N}_{a}}{|\sin \phi|} \sin ^{\frac{1}{2}} \frac{h}{2} \frac{h u(\phi)^{-\frac{2 \Delta_{\varphi}}{3}-\frac{1}{2}}}{180 \xi \sin ^{\frac{3}{2}}\left(\frac{\phi}{2}\right)} \\
& \times\left(\Gamma\left(\frac{4 \Delta_{\varphi}}{3}\right) \Gamma\left(\frac{4 \Delta_{\varphi}}{3}+2\right)\right. \\
& \times{ }_{4} F_{5}\left(\frac{2 \Delta_{\varphi}}{3}+\frac{1}{2}, \frac{2 \Delta_{\varphi}}{3}+1, \frac{2 \Delta_{\varphi}}{3}+\frac{3}{2}, \frac{2 \Delta_{\varphi}}{3} ; \frac{7}{6}, \frac{4}{3}, \frac{3}{2}, \frac{5}{3}, \frac{11}{6} ; \eta\right) \sin \left(\frac{2 \pi \Delta_{\varphi}}{3}\right) \xi^{5} \\
+ & \cos \left(\frac{1}{3}\left(2 \pi \Delta_{\varphi}+\pi\right)\right) \Gamma\left(\frac{4 \Delta_{\varphi}}{3}-\frac{1}{3}\right) \Gamma\left(\frac{4 \Delta_{\varphi}}{3}+\frac{5}{3}\right) \\
& \times{ }_{4} F_{5}\left(\frac{2 \Delta_{\varphi}}{3}-\frac{1}{6}, \frac{2 \Delta_{\varphi}}{3}+\frac{1}{3}, \frac{2 \Delta_{\varphi}}{3}+\frac{5}{6}, \frac{2 \Delta_{\varphi}}{3}+\frac{4}{3} ; \frac{5}{6}, \frac{7}{6}, \frac{4}{3}, \frac{3}{2}, \frac{5}{3} ; \eta\right) \sqrt[6]{u(\phi)} \xi^{4} \\
+ & 20\left(-\cos \left(\frac{1}{6}\left(4 \pi \Delta_{\varphi}+\pi\right)\right) \Gamma\left(\frac{4}{3}\left(\Delta_{\varphi}+1\right)\right) \Gamma\left(\frac{4 \Delta_{\varphi}}{3}-\frac{2}{3}\right)\right. \\
& \times{ }_{4} F_{5}\left(\frac{2 \Delta_{\varphi}}{3}-\frac{1}{3}, \frac{2 \Delta_{\varphi}}{3}+\frac{1}{6}, \frac{2 \Delta_{\varphi}}{3}+\frac{2}{3}, \frac{2 \Delta_{\varphi}}{3}+\frac{7}{6} ; \frac{2}{3}, \frac{5}{6}, \frac{7}{6}, \frac{4}{3}, \frac{3}{2} ; \eta\right) \sqrt[3]{u(\phi)} \xi^{3} \\
+ & \cos \left(\frac{2 \pi \Delta_{\varphi}}{3}\right) \Gamma\left(\frac{4 \Delta_{\varphi}}{3}-1\right) \Gamma\left(\frac{4 \Delta_{\varphi}}{3}+1\right)
\end{aligned}
$$




$$
\begin{aligned}
& \times{ }_{4} F_{5}\left(\frac{2 \Delta_{\varphi}}{3}-\frac{1}{2}, \frac{2 \Delta_{\varphi}}{3}+\frac{1}{2}, \frac{2 \Delta_{\varphi}}{3}+1, \frac{2 \Delta_{\varphi}}{3} ; \frac{1}{2}, \frac{2}{3}, \frac{5}{6}, \frac{7}{6}, \frac{4}{3} ; \eta\right) \sqrt{u(\phi)} \xi^{2} \\
-6 \Gamma & \left(\frac{4}{3}\left(\Delta_{\varphi}-1\right)\right) \Gamma\left(\frac{4 \Delta_{\varphi}}{3}+\frac{2}{3}\right) \\
& \times{ }_{4} F_{5}\left(\frac{2 \Delta_{\varphi}}{3}-\frac{2}{3}, \frac{2 \Delta_{\varphi}}{3}-\frac{1}{6}, \frac{2 \Delta_{\varphi}}{3}+\frac{1}{3}, \frac{2 \Delta_{\varphi}}{3}+\frac{5}{6} ; \frac{1}{3}, \frac{1}{2}, \frac{2}{3}, \frac{5}{6}, \frac{7}{6} ; \eta\right) \\
& \times(u(\phi))^{2 / 3} \sin \left(\frac{1}{3}\left(2 \pi \Delta_{\varphi}+\pi\right)\right) \xi \\
+6 \Gamma & \left(\frac{4 \Delta_{\varphi}}{3}-\frac{5}{3}\right) \Gamma\left(\frac{4 \Delta_{\varphi}}{3}+\frac{1}{3}\right) \\
& \times{ }_{4} F_{5}\left(\frac{2 \Delta_{\varphi}}{3}-\frac{5}{6}, \frac{2 \Delta_{\varphi}}{3}-\frac{1}{3}, \frac{2 \Delta_{\varphi}}{3}+\frac{1}{6}, \frac{2 \Delta_{\varphi}}{3}+\frac{2}{3} ; \frac{1}{6}, \frac{1}{3}, \frac{1}{2}, \frac{2}{3}, \frac{5}{6} ; \eta\right) \\
& \left.\left.\times(u(\phi))^{5 / 6} \sin \left(\frac{1}{6}\left(4 \pi \Delta_{\varphi}+\pi\right)\right)\right)\right)
\end{aligned}
$$

Where $\eta \equiv \frac{\xi^{6} \csc ^{2}\left(\frac{\phi}{2}\right)}{2916 h^{2}}$ and $u(\phi) \equiv-h^{2} \sin ^{2}\left(\frac{\phi}{2}\right)$. This is not a very illuminating expression, but we can see right away from the global factor at the first line that the leading singularity behaves as $1 / \xi^{d-3}$, and the other terms are polynomials in $\xi$ smoothing out the singularity. Also the argument $\eta$ in the hypergeometric functions is very small so all the hypergeometrics are essentially one. The function $u(\phi)$ is also very small. The leading term is then given by,

$$
\tilde{A}(\xi, \phi)=i \frac{(2 \pi)^{3 / 2} \mathcal{N}_{a}}{|\sin \phi|} \frac{2 h^{\frac{1-4 \Delta \varphi}{3}} \sin \left(\frac{\phi}{2}\right)^{\frac{2-4 \Delta_{\varphi}}{3}} \sin \left(\frac{1}{6}\left(4 \pi \Delta_{\varphi}+\pi\right)\right) \Gamma\left(\frac{4 \Delta_{\varphi}-5}{3}\right) \Gamma\left(\frac{4 \Delta_{\varphi}+1}{3}\right)}{3 \xi \sin ^{2}\left(\frac{\phi}{2}\right)} .
$$

We believe this correlator should be thought as a resummation of a loop ladder diagrams at the bulk point singularity of Witten diagrams with only graviton exchanges.

The reason why we expect a singularity of the form $1 / \xi^{d-3}$ for the full correlator has been explained in [32], and it comes from the fact that at the $\xi \rightarrow 0$ limit the configuration (2.7) is invariant under transformations by the group $\mathrm{SO}(1, d-2)$, and therefore the singularity should arises from the action of this non-compact group. Given the above argument is natural to believe that this singularity behavior will be present in any interacting conformal field theory. Another evidence is that the singularity is present at the level of individual conformal blocks and unless very precise cancelations occurs at the OPE expansion we might expect the singularity to survive. Let us consider quickly the case for the $O(N)$ model as a simple example of this.

The four-point function of the $O(N)$ model, has been computed at leading order in a large $N$ expansion in [50] (see also [51] for more recent analysis of anomalous dimension in the $O(N)$ model) and is given by,

$$
\left\langle\sigma_{a}\left(x_{1}\right) \sigma_{b}\left(x_{2}\right) \sigma_{c}\left(x_{3}\right) \sigma_{c}\left(x_{4}\right)\right\rangle=\frac{\eta_{1} h \Gamma(h)}{(h-2) \Gamma(h-1)^{2} N} \frac{f_{a b c d}(u, v)}{\left(x_{12}^{2} x_{34}^{2}\right)^{\Delta_{\sigma}}},
$$


with

$$
f_{a b c d}(u, v)=\delta_{a b} \delta_{c d} \bar{D}_{1, h-1,1, h-1}(u, v)+\delta_{a d} \delta_{b c} \bar{D}_{1, h-1, h-1,1}(u, v)+\delta_{a c} \delta_{b d} \bar{D}_{h-1, h-1,1,1}(u, v),
$$

and $2 h=d$. We then can use the results from appendix B [28] to extract the small $\rho$ behavior of the $D$-functions as we did in previous sections,

$$
\bar{D}_{\Delta_{1}, \Delta_{2}, \Delta_{1}, \Delta_{2}}(u, v) \sim-2 \pi^{3 / 2} \Gamma\left(\Delta_{1}+\Delta_{2}-\frac{3}{2}\right) \frac{\sigma(1-\sigma)^{\Delta_{1}+\Delta_{2}-2}}{\left(\rho^{2}\right)^{\Delta_{1}+\Delta_{2}-\frac{3}{2}}} .
$$

The first term of the four-point correlation function (4.19) at the small $\rho$ limit, looks like,

$$
\begin{aligned}
& \left\langle\sigma_{a}\left(x_{1}\right) \sigma_{b}\left(x_{2}\right) \sigma_{c}\left(x_{3}\right) \sigma_{c}\left(x_{4}\right)\right\rangle \\
& =-\frac{\eta_{1} h \Gamma(h) \Gamma\left(h-\frac{3}{2}\right)}{(h-2) \Gamma(h-1)^{2} N} \frac{2 \pi^{3 / 2}}{\left(x_{12}^{2} x_{34}^{2}\right)^{\Delta_{\sigma}}} \frac{\sigma(1-\sigma)^{h-2}}{\left(\rho^{2}\right)^{h-\frac{3}{2}}} \\
& =-\frac{\eta_{1} h \Gamma(h) \Gamma\left(h-\frac{3}{2}\right)}{(h-2) \Gamma(h-1)^{2} N} \frac{2 \pi^{3 / 2}}{\left(x_{12}^{2} x_{34}^{2}\right)^{\Delta_{\sigma}}} \frac{1}{|\sin \phi| \xi^{d-3}},
\end{aligned}
$$

displaying a singularity of the form $1 / \xi^{d-3}$ as claimed.

\section{$5 \quad$ Inverse map}

In this section we would like to invert the formula (3.5) in order to find a representation for the flat space s-matrix in terms of conformal correlation functions. In the following we are going to focus in $d=4$, but we believe the arguments can be straightforwardly generalized to other dimensions.

In order to invert equation (3.5) we are going to use the following integral,

$$
\int_{0}^{\infty} d \xi \xi K_{\frac{1}{2}}(A \xi) K_{\frac{1}{2}}(-B \xi)=-\frac{\pi}{2(A-B) \sqrt{-A B}}
$$

allowing us to write (2.18) as,

$$
\mathcal{N}_{a} \int_{0}^{\infty} d \xi \xi^{\frac{3}{2}} K_{\frac{1}{2}}(-B \xi) \tilde{A}^{(4)}(\xi, \phi)=-\frac{\pi}{2} \int_{0}^{\infty} d \Delta \frac{\Delta^{4 \Delta_{\varphi}-\frac{d}{2}-3}}{|\sin \phi|} \frac{T^{d+1}(\sqrt{s}=\Delta, \cos \phi)}{(\Delta-B) \sqrt{-B}}
$$

If we consider a polynomially bounded S-matrix, this integral is peaked around the region $\Delta \sim B$, and therefore we can approximate it as,

$$
T^{d+1}(\sqrt{s}=\Delta, \cos \phi) \sim \frac{2 i \mathcal{N}_{a}}{\pi}|\sin \phi| \Delta^{\frac{d+7}{2}-4 \Delta_{\varphi}} \int_{0}^{\infty} d \xi \xi^{\frac{3}{2}} K_{\frac{1}{2}}(-B \xi) \tilde{A}^{(4)}(\xi, \phi) .
$$

This expression can in turn be tought as a conformal field theory rederivation of PenedonesOkuda formula derived from the gravity side in [31] (see equation (2.13) there) ${ }^{8}$ Even though we were unable to proved it for general dimension, we find natural to conjecture the form of this limit at arbitrary dimensions as,

$$
T^{d+1}(\sqrt{s}=\Delta, \cos \phi) \sim \frac{2 i \mathcal{N}_{a}}{\pi}|\sin \phi| \Delta^{\frac{d+7}{2}-4 \Delta \varphi} \int_{0}^{\infty} d \xi \xi^{\frac{d-1}{2}} K_{\frac{d-3}{2}}(-B \xi) \tilde{A}^{(d)}(\xi, \phi) .
$$

\footnotetext{
${ }^{8}$ In order to compare, notice that in [31] equation (2.13), there is a global factor of $l_{s}^{3} \sqrt{s t u} \sim \Delta^{3}|\sin \phi|$.
} 
In principle, we can plug in any four-point correlation function and associate to it a flat s-matrix throughout the mapping above. However, this does not imply we will get a physically acceptable flat space s-matrix associated to a particular theory. Nevertheless, we can use this as a representation for scattering in flat space as an expansion in conformal invariant functions, regardless whether the latest is indeed a physical observable of a particular conformal field theory.

\section{Conclusions}

In this paper we have studied the bulk point singularity limit of correlation functions in generic conformal field theories. By using the fact that the conformal blocks at the bulk point singularity can be written in terms of spherical harmonics, we have proposed an expansion of four-point correlation functions in terms of a s-matrix in flat space.

We have use this mapping to transform flat-space gravitational scattering to correlation functions associated to Witten diagrams in AdS at the bulk point singularity, and shown that indeed the map reproduce the known results from direct previous calculations of Witten diagrams.

We have also shown that the eikonal resummation of gravitational scattering maps to a full correlation function with the expected behavior at the bulk point singularity that might be thought as a resummation of Witten graviton ladder exchanges around the singularity.

Finally, we have computed the inverse map transforming conformal invariant functions into flat space scattering reproducing a previous proposal computed from the gravity side in [31], providing a representation of the s-matrix as an expansion in conformal basis at the bulk singularity.

It would be nice to contrast the computations of this paper against bulk point singularity limits of higher order loop Witten diagrams perhaps by using any of the growing recent methods on the subject [52-59]

The eikonal approximation in AdS space have been thoroughly studied in a series of inspiring papers [33-37]. It would be interesting to see if there is a similar treatment for the ideas pursued in this paper.

\section{Acknowledgments}

I would like to thank to Alexander Zhiboedov and Poul H. Damgaard for enlightening discussions. This work is supported in part by the Danish National Research Foundation.

Open Access. This article is distributed under the terms of the Creative Commons Attribution License (CC-BY 4.0), which permits any use, distribution and reproduction in any medium, provided the original author(s) and source are credited.

\section{References}

[1] J.M. Maldacena, The large $N$ limit of superconformal field theories and supergravity, Int. $J$. Theor. Phys. 38 (1999) 1113 [hep-th/9711200] [INSPIRE].

[2] E. Witten, Anti-de Sitter space and holography, Adv. Theor. Math. Phys. 2 (1998) 253 [hep-th/9802150] [INSPIRE]. 
[3] J. Polchinski, $S$ matrices from AdS space-time, hep-th/9901076 [INSPIRE].

[4] L. Susskind, Holography in the flat space limit, AIP Conf. Proc. 493 (1999) 98 [hep-th/9901079] [INSPIRE].

[5] M.F. Paulos et al., The S-matrix bootstrap. Part I: QFT in AdS, JHEP 11 (2017) 133 [arXiv: 1607.06109] [INSPIRE].

[6] M.F. Paulos et al., The S-matrix bootstrap. Part II: two dimensional amplitudes, JHEP 11 (2017) 143 [arXiv: 1607.06110] [INSPIRE].

[7] A.L. Fitzpatrick and J. Kaplan, Scattering states in AdS/CFT, arXiv:1104.2597 [INSPIRE].

[8] J. Penedones, Writing CFT correlation functions as AdS scattering amplitudes, JHEP 03 (2011) 025 [arXiv: 1011.1485] [INSPIRE].

[9] A.L. Fitzpatrick et al., A natural language for AdS/CFT correlators, JHEP 11 (2011) 095 [arXiv:1107.1499] [INSPIRE].

[10] A.L. Fitzpatrick and J. Kaplan, Analyticity and the Holographic S-matrix, JHEP 10 (2012) 127 [arXiv: 1111.6972] [INSPIRE].

[11] A.L. Fitzpatrick and J. Kaplan, Unitarity and the holographic S-matrix, JHEP 10 (2012) 032 [arXiv: 1112.4845] [inSPIRE].

[12] D. Nandan, A. Schreiber, A. Volovich and M. Zlotnikov, Celestial amplitudes: conformal partial waves and soft limits, JHEP 10 (2019) 018 [arXiv: 1904.10940] [INSPIRE].

[13] E. Hijano, Semi-classical BMS 3 blocks and flat holography, JHEP 10 (2018) 044 [arXiv: 1805.00949] [INSPIRE].

[14] E. Hijano, Flat space physics from AdS/CFT, JHEP 07 (2019) 132 [arXiv: 1905.02729] [INSPIRE].

[15] H. Bondi, M.G.J. van der Burg and A.W.K. Metzner, Gravitational waves in general relativity. 7. Waves from axisymmetric isolated systems, Proc. Roy. Soc. Lond. A 269 (1962) 21.

[16] R. Sachs, Asymptotic symmetries in gravitational theory, Phys. Rev. 128 (1962) 2851 [INSPIRE].

[17] S. Pasterski, S.-H. Shao and A. Strominger, Flat space amplitudes and conformal symmetry of the celestial sphere, Phys. Rev. D 96 (2017) 065026 [arXiv:1701.00049] [INSPIRE].

[18] C. Cheung, A. de la Fuente and R. Sundrum, 4D scattering amplitudes and asymptotic symmetries from $2 D$ CFT, JHEP 01 (2017) 112 [arXiv:1609.00732] [INSPIRE].

[19] A. Ball et al., Uplifting AdS $S_{3} / C F T_{2}$ to flat space holography, JHEP 08 (2019) 168 [arXiv: 1905.09809] [INSPIRE].

[20] C. Cardona and Y.-t. Huang, S-matrix singularities and CFT correlation functions, JHEP 08 (2017) 133 [arXiv: 1702.03283] [INSPIRE].

[21] S. Pasterski and S.-H. Shao, Conformal basis for flat space amplitudes, Phys. Rev. D 96 (2017) 065022 [arXiv: 1705.01027] [INSPIRE].

[22] S. Pasterski, S.-H. Shao and A. Strominger, Gluon amplitudes as $2 d$ conformal correlators, Phys. Rev. D 96 (2017) 085006 [arXiv: 1706. 03917] [InSPIRE].

[23] H.T. Lam and S.-H. Shao, Conformal basis, optical theorem and the bulk point singularity, Phys. Rev. D 98 (2018) 025020 [arXiv:1711.06138] [INSPIRE].

[24] S. Banerjee and P. Pandey, Conformal properties of soft operators - 2: use of null-states, arXiv: 1906.01650 [INSPIRE]. 
[25] T. Adamo, L. Mason and A. Sharma, Celestial amplitudes and conformal soft theorems, Class. Quant. Grav. 36 (2019) 205018 [arXiv:1905.09224] [INSPIRE].

[26] S. Stieberger and T.R. Taylor, Symmetries of celestial amplitudes, Phys. Lett. B 793 (2019) 141 [arXiv: 1812.01080] [INSPIRE].

[27] A. Schreiber, A. Volovich and M. Zlotnikov, Tree-level gluon amplitudes on the celestial sphere, Phys. Lett. B 781 (2018) 349 [arXiv:1711.08435] [INSPIRE].

[28] M. Gary, S.B. Giddings and J. Penedones, Local bulk S-matrix elements and CFT singularities, Phys. Rev. D 80 (2009) 085005 [arXiv: 0903.4437] [INSPIRE].

[29] M. Gary and S.B. Giddings, The flat space S-matrix from the AdS/CFT correspondence?, Phys. Rev. D 80 (2009) 046008 [arXiv: 0904.3544 ] [inSPIRE].

[30] I. Heemskerk, J. Penedones, J. Polchinski and J. Sully, Holography from conformal field theory, JHEP 10 (2009) 079 [arXiv:0907.0151] [INSPIRE].

[31] T. Okuda and J. Penedones, String scattering in flat space and a scaling limit of Yang-Mills correlators, Phys. Rev. D 83 (2011) 086001 [arXiv: 1002.2641] [INSPIRE].

[32] J. Maldacena, D. Simmons-Duffin and A. Zhiboedov, Looking for a bulk point, JHEP 01 (2017) 013 [arXiv:1509.03612] [INSPIRE].

[33] L. Cornalba, M.S. Costa, J. Penedones and R. Schiappa, Eikonal Approximation in AdS/CFT: From Shock Waves to Four-Point Functions, JHEP 08 (2007) 019 [hep-th/0611122] [INSPIRE].

[34] L. Cornalba, M.S. Costa, J. Penedones and R. Schiappa, Eikonal approximation in AdS/CFT: conformal partial waves and finite $N$ four-point functions, Nucl. Phys. B 767 (2007) 327 [hep-th/0611123] [INSPIRE].

[35] L. Cornalba, M.S. Costa and J. Penedones, Eikonal approximation in AdS/CFT: resumming the gravitational loop expansion, JHEP 09 (2007) 037 [arXiv:0707.0120] [INSPIRE].

[36] L. Cornalba, M.S. Costa and J. Penedones, Eikonal methods in AdS/CFT: BFKL Pomeron at weak coupling, JHEP 06 (2008) 048 [arXiv:0801.3002] [INSPIRE].

[37] L. Cornalba, Eikonal methods in AdS/CFT: Regge theory and multi-reggeon exchange, arXiv:0710.5480 [INSPIRE].

[38] M. Kulaxizi, A. Parnachev and A. Zhiboedov, Bulk phase shift, CFT Regge limit and Einstein gravity, JHEP 06 (2018) 121 [arXiv:1705.02934] [INSPIRE].

[39] Z. Komargodski and A. Zhiboedov, Convexity and liberation at large spin, JHEP 11 (2013) 140 [arXiv: 1212.4103] [INSPIRE].

[40] A.L. Fitzpatrick, J. Kaplan, D. Poland and D. Simmons-Duffin, The analytic bootstrap and AdS superhorizon locality, JHEP 12 (2013) 004 [arXiv:1212.3616] [INSPIRE].

[41] A.L. Fitzpatrick, E. Katz, D. Poland and D. Simmons-Duffin, Effective conformal theory and the flat-space limit of AdS, JHEP 07 (2011) 023 [arXiv: 1007.2412] [INSPIRE].

[42] E. D'Hoker et al., Graviton exchange and complete four point functions in the AdS/CFT correspondence, Nucl. Phys. B 562 (1999) 353 [hep-th/9903196] [INSPIRE].

[43] E. D'Hoker, D.Z. Freedman and L. Rastelli, AdS/CFT four point functions: how to succeed at $z$ integrals without really trying, Nucl. Phys. B 562 (1999) 395 [hep-th/9905049] [INSPIRE].

[44] A. Koemans Collado, P. Di Vecchia and R. Russo, Revisiting the second post-Minkowskian eikonal and the dynamics of binary black holes, Phys. Rev. D 100 (2019) 066028 [arXiv: 1904.02667] [INSPIRE]. 
[45] M. Levy and J. Sucher, Eikonal approximation in quantum field theory, Phys. Rev. 186 (1969) 1656 [INSPIRE].

[46] D. Amati, M. Ciafaloni and G. Veneziano, Can space-time be probed below the string size?, Phys. Lett. B 216 (1989) 41 [InSPIRE].

[47] D. Amati, M. Ciafaloni and G. Veneziano, Superstring collisions at Planckian energies, Phys. Lett. B 197 (1987) 81 [INSPIRE].

[48] I.J. Muzinich and M. Soldate, High-energy unitarity of gravitation and strings, Phys. Rev. D 37 (1988) 359 [INSPIRE].

[49] S.B. Giddings and M. Srednicki, High-energy gravitational scattering and black hole resonances, Phys. Rev. D 77 (2008) 085025 [arXiv:0711.5012] [INSPIRE].

[50] K. Lang and W. Rühl, The critical $O(N) \sigma$-model at dimension $2<d<4$ and order $1 / n^{2}$ : operator product expansions and renormalization, Nucl. Phys. B 377 (1992) 371 [INSPIRE].

[51] L.F. Alday and A. Zhiboedov, An algebraic approach to the analytic bootstrap, JHEP 04 (2017) 157 [arXiv: 1510.08091] [INSPIRE].

[52] C. Cardona, Mellin-(Schwinger) representation of One-loop Witten diagrams in AdS, arXiv: 1708.06339 [INSPIRE].

[53] L.F. Alday, A. Bissi and E. Perlmutter, Genus-one string amplitudes from conformal field theory, JHEP 06 (2019) 010 [arXiv: 1809.10670] [INSPIRE].

[54] L.F. Alday and A. Bissi, Loop corrections to supergravity on $A d S_{5} \times S^{5}$, Phys. Rev. Lett. 119 (2017) 171601 [arXiv:1706.02388] [INSPIRE].

[55] O. Aharony, L.F. Alday, A. Bissi and E. Perlmutter, Loops in AdS from conformal field theory, JHEP 07 (2017) 036 [arXiv: 1612.03891] [InSPIRE].

[56] I. Bertan and I. Sachs, Loops in Anti-de Sitter space, Phys. Rev. Lett. 121 (2018) 101601 [arXiv: 1804.01880] [INSPIRE].

[57] E.Y. Yuan, Simplicity in AdS perturbative dynamics, arXiv:1801.07283 [INSPIRE].

[58] F. Aprile, J.M. Drummond, P. Heslop and H. Paul, Loop corrections for Kaluza-Klein AdS amplitudes, JHEP 05 (2018) 056 [arXiv:1711.03903] [INSPIRE].

[59] S. Giombi, C. Sleight and M. Taronna, Spinning AdS loop diagrams: two point functions, JHEP 06 (2018) 030 [arXiv: 1708.08404] [INSPIRE]. 